\title{
Commentary
}

\section{Clearance Prediction Methodology Needs Fundamental Improvement: Trends Common to Rat and Human Hepatocytes/Microsomes and Implications for Experimental Methodology}

\author{
F. L. Wood, ${ }^{1}$ J. B. Houston, and D. Hallifax \\ Centre for Applied Pharmacokinetic Research, Division of Pharmacy and Optometry, \\ School of Health Sciences, Faculty of Biology, Medicine and Health, University of \\ Manchester, Manchester Academic Health Science Centre, Manchester, United Kingdom
}

Received June 13, 2017; accepted September 6, 2017

\begin{abstract}
Although prediction of clearance using hepatocytes and liver microsomes has long played a decisive role in drug discovery, it is widely acknowledged that reliably accurate prediction is not yet achievable despite the predominance of hepatically cleared drugs. Physiologically mechanistic methodology tends to underpredict clearance by several fold, and empirical correction of this bias is confounded by imprecision across drugs. Understanding the causes of prediction uncertainty has been slow, possibly reflecting poor resolution of variables associated with donor source and experimental methods, particularly for the human situation. It has been reported that among published human hepatocyte predictions there was a tendency for underprediction to increase with increasing in vivo intrinsic clearance, suggesting an inherent limitation using this particular system. This
\end{abstract}

implied an artifactual rate limitation in vitro, although preparative effects on cell stability and performance were not yet resolved from assay design limitations. Here, to resolve these issues further, we present an up-to-date and comprehensive examination of predictions from published rat as well as human studies (where $n=128$ and 101 hepatocytes and $n=71$ and 83 microsomes, respectively) to assess system performance more independently. We report a clear trend of increasing underprediction with increasing in vivo intrinsic clearance, which is similar both between species and between in vitro systems. Hence, prior concerns arising specifically from human in vitro systems may be unfounded and the focus of investigation in the future should be to minimize the potential in vitro assay limitations common to whole cells and subcellular fractions.

\section{Introduction}

For more than a decade numerous studies have reported prediction of clearance of drugs from human and rat hepatic in vitro systems (with increasing emphasis on hepatocytes rather than microsomes), all of which have indicated a tendency for underprediction based on physiologically mechanistic scaling (Shibata et al., 2002; Hallifax et al., 2005, 2010; Ito and Houston, 2005; Riley et al., 2005; Brown et al., 2007; Stringer et al., 2008; Chiba et al., 2009). These studies used both intra- and interlaboratory data sets for drugs that were mostly commercially available small molecules (200-600 mol. wt.) with a predominant metabolic route of clearance. With an increasing

This work was funded by the Centre for Applied Pharmacokinetic Research consortium membership, which included GSK, Janssen, Lilly, and Pfizer.

${ }^{1}$ Current affiliation: The Institute of Cancer Research, Sutton, United Kingdom. https://doi.org/10.1124/dmd.117.077040. emphasis on human (hepatic) in vitro systems, in vivo intrinsic clearance $\left(\mathrm{CL}_{\mathrm{int}}\right)$ of these drugs ranged over about five orders of magnitude $(1-100,000 \mathrm{ml} / \mathrm{min} / \mathrm{kg})$.

To limit the negative impact of prediction inaccuracy on drug discovery and human dosing decisions, empirical correction of prediction bias, as has been suggested, offers a practical and justifiable improvement (Poulin et al., 2012; Sohlenius-Sternbeck et al., 2012; Yamagata et al., 2017). However, considerable uncertainty remains, reflecting the poor precision that accompanies the bias seen in published data sets. Reducing both the imprecision and bias requires understanding their causes, and for prediction methodology to progress a mechanistic approach must be maintained and improved. There have been a number of suggested potential causes of underprediction, but many have not been met with incisive investigation.

The impact of the liver model choice is still questioned despite demonstration of marginal effect between the well-stirred and parallel tube models for a large data set (Hallifax et al., 2010). Only very high

ABBREVIATIONS: AFE, average fold error; BDDCS, biopharmaceutics drug disposition classification system; $\mathrm{CL}_{b}$, blood clearance; $C L_{h}$, hepatic clearance; $\mathrm{CL}_{\text {int }}$, intrinsic clearance; $\mathrm{CL}_{\text {int,u }}$, unbound intrinsic clearance; $\mathrm{ESF}$, empirical scaling factor; fu, fraction unbound; HLM, human liver microsomes; P450, cytochrome P450; $\mathrm{R}_{\mathrm{b}}$, blood/plasma concentration ratio; RLM, rat liver microsomes; RMSE, root-mean-square error; UGT, uridine $5^{\prime}$-diphospho-glucuronosyltransferase. 
clearance compounds are significantly impacted and those are inevitably a minor proportion of a typical data set. While the modeling of hepatic clearance has been extended to include transmembrane processes (Chiba et al., 2009), this is more applicable to more recently developed, less permeable drugs, and in any case cannot be as widely assessed due to the general lack of distinction of uptake processes in available in vitro data. Related to liver models, the extent of protein binding is often cited as a factor in prediction accuracy, possibly due to perceived inherent binding measurement inaccuracy, a lack of equilibrium in vivo, or facilitated uptake of drug (Soars et al., 2007b; Ring et al., 2011; Poulin et al., 2012; Bowman and Benet, 2016). For highly lipophilic drugs, Poulin et al. (2012) and Poulin and Haddad (2013) proposed a methodology relating plasma and hepatic albumin binding based on postulated involvement of bound drug with the uptake process. However, this semimechanistic methodology has been shown to offer no improvement on the conventional physiologic approach (Yamagata et al., 2017).

Human hepatocytes are inherently variable in drug clearance capability in vitro, reflecting a number of unavoidable factors: donor phenotypic variability, potentially detrimental processing (preparation and storage), and lability of metabolizing enzymes and uptake transporters-all sources of variation and possibly bias (Hallifax and Houston, 2009). Prediction assay methodology itself, lacking in standardization, is probably another source of variation (and bias); given the extremely wide range of in vivo $\mathrm{CL}_{\text {int }}$ involved, system artifacts might be expected. Addressing such issues, Hallifax et al. (2010) highlighted a tendency for prediction accuracy from human hepatocytes (but not microsomes) to decrease with increasing in vivo $\mathrm{CL}_{\mathrm{int}}$ among an extensive data set from published studies, suggesting in vitro artifacts (e.g., cofactor exhaustion) or in vitro permeability rate limitation to explain underprediction. A lack of relationship between prediction accuracy and permeability was later demonstrated (Hallifax et al., 2012), focusing attention on assay methodology. In a recent comparison of published studies using human hepatocytes and microsomes, Bowman and Benet (2016) saw only slight evidence of difference in prediction accuracy between drugs classified [biopharmaceutics drug disposition classification system (BDDCS)] as transporter substrates or not, or between drugs segregated as highly bound in blood or not. Having confirmed the lack of resolution between sources of uncertainty, Bowman and Benet (2016) highlighted a need for improved experimental methodology.

Use of rat in vitro systems for measurement of $\mathrm{CL}_{\text {int }}$ has been superseded by the now widely available human equivalent. Although some studies have indicated a tendency toward underprediction of clearance for rat as well as that more widely acknowledged for human (Huang et al., 2010; Sohlenius-Sternbeck et al., 2012), minimal interindividual and processing variability in rat implies much less prediction uncertainty (Iwatsubo et al., 1996, 1997; Wilson et al., 2003; Ito and Houston, 2005; Riley et al., 2005; Hallifax and Houston, 2009). Therefore, it is of considerable potential value to appraise the limitations of clearance prediction in rat, in parallel to human, to enable more incisive understanding of experimental uncertainty. Going beyond previous assessments of prediction of clearance and to attempt to resolve source-based and experimentally based variation and bias, we have compiled the most extensive data sets to date for interspecies (rat and human) comparison of in vitro (hepatocyte and microsome) predictions of $\mathrm{CL}_{\text {int }}$ from the literature. This commentary critically examines the accuracy, precision, and trends in prediction of clearance between the foundational in vitro species, assessing the implications for future progress in prediction methodology.

\section{Data Collation and Analysis}

Data sets for human ( $n=101$, hepatocytes; $n=83$, microsomes) and rat ( $n=128$ hepatocytes; $n=71$, microsomes) in vitro $\mathrm{CL}_{\text {int }}$ and in vivo clearance were compiled based on examination of existing published data sets and a further search of the literature. Both approved pharmaceuticals and investigatory proprietary compounds were considered provided that complementary in vitro and in vivo data were available. Criteria for inclusion of in vivo data were that reported blood clearance $\left(\mathrm{CL}_{\mathrm{b}}\right)$ or plasma clearance was determined from intravenous dosing and that $\mathrm{CL}_{\mathrm{b}}$ did not exceed hepatic blood flow $\left(Q_{\mathrm{h}}\right)$. In vitro $\mathrm{CL}_{\text {int }}$ determined from either metabolite formation or substrate depletion over a range of substrate concentrations, or from single concentration substrate depletion time profiles was considered. In vitro data included both suspended hepatocytes and liver microsomes, reflecting the prevalent use of these systems in the pharmaceutical industry. Microsomal data represent that from incubations with exogenous NADPH (phase I metabolism) only. Hepatocyte data represent incubations conducted in the absence of serum; in each instance the use of fresh or cryopreserved cells was recorded.

For human hepatocyte data, two key review articles, Hallifax et al. (2010) and Paixao et al. (2010), were identified; from these the original sources were examined and data not previously included were added [for e.g., Hallifax et al. (2010) included only predictions from cryopreserved hepatocytes]. Most additional data came from subsequently published studies (Sohlenius-Sternbeck et al., 2010, 2012; Akabane et al., 2012,b); some data came from earlier studies not previously included (Hallifax et al., 2005, 2008). For human liver microsomes (HLM), original sources from Ito and Houston (2004) and Hallifax et al. (2010) were examined, with supplementation from Obach (1999), Cubitt et al. (2009), Gertz et al. (2010), and Sohlenius-Sternbeck et al. (2010). Additional data for both human hepatocyte and HLM were provided by Stringer (2006) as data supplemental to Stringer et al. (2008). The rat hepatocyte data sets encompassed a previous compilation (Ito and Houston, 2005) and several subsequent original research articles; the majority of data were from Huang et al. (2010) and Sohlenius-Sternbeck et al. (2010). For rat liver microsomes (RLM), original source data cited by Houston (1994), Houston and Carlile (1997), and Ito and Houston (2004) were collated together with data from Jones and Houston (2004), De Buck et al. (2007), and Huang et al. (2010). The complete data sets (with sources) are given in supplemental table 1 (human) and supplemental table 2 (rat). No data were duplicated by source; where more than one original source provided data for a particular drug, mean $\mathrm{CL}_{\text {int }}$ was calculated.

The data sets were considered to predominantly comprise highly permeable drugs of which the vast majority would be expected to be cleared by metabolism without rate limitation by transport, although dependency on hepatic uptake transport for clearance would not necessarily preclude inclusion of predictions for the purposes of this analysis. Varma et al. (2015) proposed a drug classification system for predicting the major clearance route based on charge, permeability, and molecular weight, i.e., the extended clearance classification system. Using the assignments they presented, the human data sets in the present study (as far as could be assigned: $73 \%$ and $72 \%$ hepatocytes and microsomes, respectively) comprised about $86 \%$ deemed to have metabolism as the primary route of clearance-which supports our initial assumption. Of the remainder, half (7\%) could be assigned as rate limited by hepatic uptake transport and half (7\%) dependent on renal clearance. For rat, about $79 \%$ and $100 \%$ (hepatocytes and microsomes, respectively) of the commercial drugs that were extended clearance classification system precategorized $(60 \%$ and $65 \%$ of commercial drugs for hepatocytes and microsomes, respectively) were predominantly cleared by metabolism. A large proportion of the rat data sets were proprietary compounds; however, based on their physicochemical properties, at least $85 \%$ for hepatocytes and $100 \%$ for microsomes [assuming all AZ-designated compounds were highly permeable as denoted by Varma et al. (2015), i.e., $>5 \times 10^{6} \mathrm{~cm} / \mathrm{s}$ ] were inferred as 
predominantly cleared by metabolism. Since the aforementioned categorization is inherently imprecise, no particular individual drug prediction was excluded following the initial collation criteria.

In Vitro Fraction Unbound. In studies where the fraction unbound (fu) in either microsomes $\left(\mathrm{fu}_{\mathrm{mic}}\right)$ or hepatocytes ( $\left.\mathrm{fu}_{\mathrm{heps}}\right)$ was experimentally determined and reported alongside $\mathrm{CL}_{\text {int }}$, this value was applied in prediction of in vivo unbound $\mathrm{CL}_{\text {int }}\left(\mathrm{CL}_{\mathrm{int}, \mathrm{u}}\right)$; where $\mathrm{fu}_{\text {mic }}$ or $\mathrm{fu}_{\text {heps }}$ were unreported, these values were estimated using the lipophilicity relationship algorithms given by eq. 1 (Hallifax and Houston, 2006) and eq. 2 (Kilford et al., 2008), respectively,

$$
\begin{aligned}
\mathrm{fu}_{\text {mic }} & =\frac{1}{1+\mathrm{P} \cdot 10^{0.072 \cdot \log P / D^{2}+0.067 \cdot \log P / D-1.126}} \\
\mathrm{fu}_{\text {heps }} & =\frac{1}{1+125 \cdot \mathrm{V}_{\mathrm{R}} \cdot 10^{0.072 \cdot \log P / D^{2}+0.067 \cdot \log P / D-1.126}}
\end{aligned}
$$

where $\mathrm{P}$ is the microsomal protein concentration; $\log P / D$ is either the $\log P$ value for basic and neutral drugs or the $\log D$ value for acidic drugs; and $\mathrm{V}_{\mathrm{R}}$ is the volume ratio of hepatocytes to medium ( 0.005 for $1 \times 10^{6}$ cells $/ \mathrm{ml}$ ) (Brown et al., 2007).

Scaling In Vitro Intrinsic Clearance to Whole Liver. In vitro $\mathrm{CL}_{\text {int }}$ values were scaled to the in vivo whole liver equivalent using eq. 3 (Hallifax et al., 2010), where the physiologically based scaling factor (PBSF) is the microsomal average recovery factor for microsomal predictions and hepatocellularity for hepatocyte predictions, and LW is the liver weight $/ \mathrm{kg}$ body weight.

$$
\text { Predicted in vivo } \mathrm{CL}_{\mathrm{int}, \mathrm{u}}=\frac{\text { in vitro } \mathrm{CL}_{\mathrm{int}} \times \mathrm{PBSF} \times \mathrm{LW}}{\mathrm{fu}_{\mathrm{mic}} \text { or } \mathrm{fu}_{\mathrm{heps}}}
$$

Microsomal recovery factors of $40 \mathrm{mg}$ microsomal protein/g liver (Hakooz et al., 2006) and $60 \mathrm{mg}$ microsomal protein/g liver (Houston and Carlile, 1997) were used for human and rat, respectively; hepatocellularity of $120 \times 10^{6}$ hepatocytes/g liver was used for both human (Hakooz et al., 2006) and rat (Bayliss et al., 1999) and the liver weight was $21.4 \mathrm{~g} / \mathrm{kg}$ body weight for human and $40 \mathrm{~g} / \mathrm{kg}$ body weight for rat (Davies and Morris, 1993).

Determination of In Vivo Intrinsic Clearance. In vivo $\mathrm{CL}_{\mathrm{int}, \mathrm{u}}$ was derived from hepatic clearance $\left(\mathrm{CL}_{\mathrm{h}}\right)$ (blood), fraction unbound in blood $\left(\mathrm{fu}_{\mathrm{b}}\right)$ and blood flow $\left(Q_{\mathrm{h}}\right)$ with a given value of $20.7 \mathrm{ml} / \mathrm{min} / \mathrm{kg}$ for human (Davies and Morris, 1993) and $100 \mathrm{ml} / \mathrm{min} / \mathrm{kg}$ for rat (Ito and Houston, 2004). Where applicable and data available, $\mathrm{CL}_{\mathrm{h}}$ was determined by subtracting renal clearance from total $\mathrm{CL}_{\mathrm{b}}$. Where sources provided the plasma clearance and fraction unbound in plasma $\left(\mathrm{fu}_{\mathrm{p}}\right), \mathrm{CL}_{\mathrm{b}}$ and $\mathrm{fu}_{\mathrm{b}}$ were calculated using the reported blood/plasma concentration ratio $\left(R_{b}\right)$, i.e., plasma clearance/ $R_{b}$ and $f_{p} / R_{b}$, respectively. Where $R_{b}$ was unavailable, it was assumed to be equal to 1 for a basic or neutral compound and 0.55 (1-hematocrit) for an acidic compound. If data from multiple studies were available for the same compound, the arithmetic means of $\mathrm{CL}_{\mathrm{b}}$ and $\mathrm{fu}_{\mathrm{b}}$ were used in the calculation of $\mathrm{CL}_{\mathrm{int}, \mathrm{u}}$.

In vivo $\mathrm{CL}_{\text {int,u }}$ was derived using both the well-stirred and parallel tube models of hepatic clearance to assess the impact of the liver model on the predictive accuracy of the in vitro data. Since the difference in bias between these two liver models (representing both extremes of drug hepatic dispersion) was found to be marginal, consistent with previous studies (Jones and Houston, 2004; Riley et al., 2005; Brown et al., 2007), data from the well-stirred model (eq. 4) are presented.

$$
\text { In vivo } \mathrm{CL}_{\mathrm{int}, \mathrm{u}}=\frac{\mathrm{CL}_{\mathrm{h}}}{\mathrm{fu}_{\mathrm{b}} \times\left[1-\left(\mathrm{CL}_{\mathrm{h}} / Q_{\mathrm{h}}\right)\right]}
$$

Assessment of Accuracy and Precision of Predictions. The overall bias in predictions was assessed by calculation of the average fold error
(AFE) (eq. 5). The root-mean-square error (RMSE) (eq. 6) was used as a measure of precision,

$$
\begin{aligned}
\mathrm{AFE} & =10\left[\sum \log (\text { predicted } / \text { observed })\right] / n \\
\mathrm{RMSE} & =\sqrt{\frac{1}{n} \sum(\text { predicted }- \text { observed })^{2}}
\end{aligned}
$$

where $n=$ number of predictions. Since underprediction yields an AFE below 1, underprediction was also expressed as fold underprediction (inverse of AFE). The percentage of $\mathrm{CL}_{\mathrm{int}, \mathrm{u}}$ predictions within (and beyond) 2-fold of in vivo was used as an additional indicator of predictive accuracy, which was consistent with previous publications (Obach, 1999; Naritomi et al., 2001; McGinnity et al., 2004; Stringer et al., 2008; Sohlenius-Sternbeck et al., 2012; Chan et al., 2013).

Calculation of Empirical Scaling Factors. The empirical scaling factors (ESFs) required to equate predicted $\mathrm{CL}_{\mathrm{int}, \mathrm{u}}$ with observed $\mathrm{CL}_{\mathrm{int}, \mathrm{u}}$ for individual compounds within each data set were calculated using eq. 7

$$
\mathrm{ESF}=\frac{\text { observed } \mathrm{CL}_{\mathrm{int}, \mathrm{u}}}{\text { predicted } \mathrm{CL}_{\mathrm{int}, \mathrm{u}}}
$$

The average ESF (for $\mathrm{CL}_{\mathrm{int}, \mathrm{u}}$ and $\mathrm{fu}_{\mathrm{p}}$ subsets) was calculated using eq. 8 (log average)

$$
\text { Average ESF }=10\left[\sum^{[\log (\text { observed } / \text { predicted })] / n}\right.
$$

Segregation of Predictions into Subsets. For the human data set, hepatocyte predictions were segregated into those derived from freshly isolated $(n=52)$ or cryopreserved cells $(n=93)$ to enable comparison of predictive accuracy between such preparations. Although several original research studies have performed such a comparison on small numbers of drugs, in which few significant differences were found (Diener et al., 1995; Li et al., 1999; Hewitt et al., 2000; Lau et al., 2002; Naritomi et al., 2003; McGinnity et al., 2004; Blanchard et al., 2005; Floby et al., 2009), this compilation provided an opportunity to address this question on a larger scale. To eliminate any bias associated with the inclusion of different substrates, a data set comprised of only compounds common to both fresh and cryopreserved hepatocyte predictions $(n=43)$ was also evaluated.

A recent review by Jones et al. (2015) indicated that the pharmaceutical industry has less confidence in predictions of noncytochrome P450mediated clearance predictions than their cytochrome P450-mediated counterparts. To test this assumption, human hepatocyte predictions of substrates for uridine 5' -diphospho-glucuronosyltransferases (UGTs), the major non-P450 metabolic enzyme family, were segregated from the remainder of the data set (predominantly P450 substrates). Glucuronidated compounds were identified and categorized by fraction metabolized by UGT based on published in vitro and in vivo data (Miners and Mackenzie, 1991; Kaiser et al., 1992; Laethem et al., 1995; Soars et al., 2002).

Prompted by the recent publication by Bowman and Benet (2016), the relationship between protein binding and accuracy of in vivo clearance predictions (from both human hepatocytes and HLM) was also reassessed. In this study, the $\mathrm{fu}_{\mathrm{p}}$, as opposed to the $\mathrm{fu}_{\mathrm{b}}$, was used as the measure of protein binding to circumvent the influence of drug binding to red blood cells. In addition, consistent with Bowman and Benet (2016), drugs were segregated by BDDCS class to examine potential relationships between drugs identified as substrates of uptake or efflux transporters, protein binding, and underprediction (human). For human hepatocyte and HLM predictions, 99/101 and 83/83 drugs, respectively, were able to be classified by BDDCS according to Benet et al. (2011) and Hosey et al. (2016). In addition, the BDDCS 
assignments were used to distinguish drug type for the analysis of ESF (both species). For rat, 125/128 (hepatocytes) and 65/71 (RLM) assignments were made including the proprietary compounds based on our assessment of the actual/likely physicochemical properties. To support the examination of drug properties, drugs (human and rat hepatocyte data sets) were identified as acidic, basic, or neutral.

\section{Comparison of Human and Rat In Vitro Prediction of Intrinsic Clearance}

Using the criteria detailed previously, predictions of in vivo $\mathrm{CL}_{\mathrm{int}, \mathrm{u}}$ were recorded for 101 drugs from human hepatocyte data (Fig. 1A) and for 83 drugs from HLM data (Fig. 1B); 66 drugs were common to both systems in human. In vivo $\mathrm{CL}_{\text {int, }}$ predictions for 128 compounds were made from rat hepatocyte data (Fig. 1C) and for 71 compounds from RLM data (Fig. 1D); 52 compounds were common to both systems in rat. In vivo and in vitro clearance data and references for individual compounds are given in the supplement (Tables 1 and 2 for human and rat, respectively).

In vivo $\mathrm{CL}_{\text {int,u }}$ was predominantly underpredicted in hepatocytes and microsomes for both human and rat. In both species underprediction was greatest in hepatocytes with an average fold underprediction of 4.2 in human and 4.7 in rat; microsomes showed less overall bias with average fold underprediction of 2.8 in human and 2.3 in rat (Table 1). However, despite evidence of less average bias in microsomes, the percentage of predictions within 2-fold of observed was similar across systems and species, ranging between $20 \%$ and $30 \%$. In both species, microsomes showed a greater incidence of overprediction (predicted values more than 2-fold above observed) than hepatocytes. Precision as represented by RMSE was similar between human hepatocytes and HLM; precision for rat was much less than for human, least of all for RLM (Table 1).

In both species, microsomes appear to display a wider range of $\mathrm{CL}_{\mathrm{int}, \mathrm{u}}$ predictions than hepatocytes. In human, predicted $\mathrm{CL}_{\mathrm{int}, \mathrm{u}}$ in hepatocytes ranged from approximately $1-1000 \mathrm{ml} / \mathrm{min} / \mathrm{kg}$ compared with approximately $0.1-10,000 \mathrm{ml} / \mathrm{min} / \mathrm{kg}$ in microsomes (Fig. 1, A and B, respectively). In rat, predicted $\mathrm{CL}_{\mathrm{int}, \mathrm{u}}$ appears to be limited to approximately $10,000 \mathrm{ml} / \mathrm{min} / \mathrm{kg}$ in hepatocytes, but reaches $100,000 \mathrm{ml} / \mathrm{min} / \mathrm{kg}$ in microsomes (Fig. 1, C and D, respectively). The greater imprecision implied by RMSE for rat may have at least in part reflected the greater upper range (hence greater error) in this species. An apparent intercept of prediction trend at the line of unity occurred at about $10 \mathrm{ml} / \mathrm{min} / \mathrm{kg}$ for human and about $100 \mathrm{ml} / \mathrm{min} / \mathrm{kg}$ for rat, possibly reflecting the general difference in metabolic rate expected between these two species.

\section{Comparison of Predictions from Fresh and Cryopreserved Human Hepatocytes}

Division of the complete human hepatocyte data set into in vivo $\mathrm{CL}_{\mathrm{int}, \mathrm{u}}$ predictions from fresh and cryopreserved preparations revealed no meaningful differences in both prediction bias and precision (Table 2). This finding was consolidated by the similar outcome from analysis of only those drugs $(n=43)$ common to both types of preparation (Fig. 2; Table 2). Therefore, there was clear evidence that the cryopreservation process has no influence on prediction accuracy.
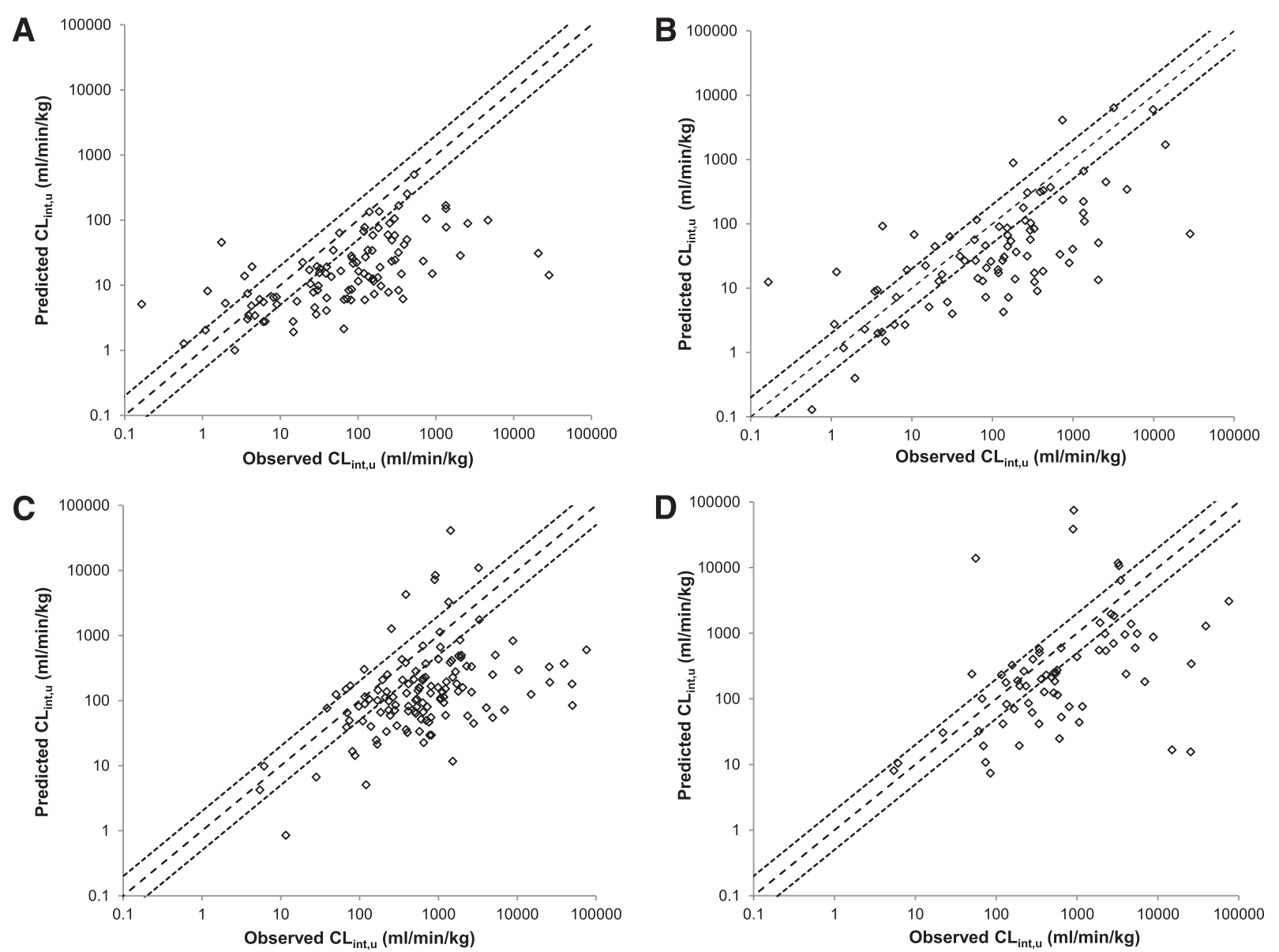

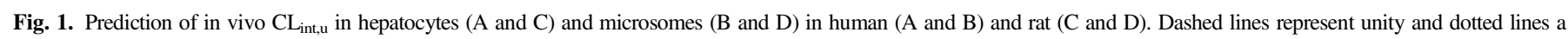

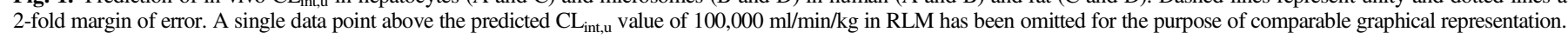


TABLE 1

Accuracy and precision of in vivo $\mathrm{CL}_{\mathrm{int}, \mathrm{u}}$ predictions in human and rat hepatocytes and liver microsomes

Data represented by AFE, average fold underprediction, RMSE, and percentage of predictions that fall within, above, and below 2-fold of observed in vivo $C L_{\text {int }, u} ; n=$ number of drugs.

\begin{tabular}{lccccc}
\hline \multirow{2}{*}{ Parameter } & \multicolumn{2}{c}{ Human } & & \multicolumn{2}{c}{ Rat } \\
\cline { 2 - 3 } \cline { 5 - 6 } & Hepatocytes & Microsomes & & Hepatocytes & Microsomes \\
\hline$n$ & 101 & 83 & & 128 & 71 \\
AFE & 0.24 & 0.36 & & 0.21 & 0.43 \\
Average fold underprediction & 4.2 & 2.8 & & 4.7 & 2.3 \\
RMSE & 3548 & 3524 & 36,203 & 63,280 \\
Predictions within 2-fold of observed (\%) & 24 & 25 & & 20 & 30 \\
Predictions more than 2-fold above observed (\%) & 7 & 69 & & 14 \\
Predictions more than 2-fold below observed (\%) & 69 & 60 & & 71 & 56 \\
\hline
\end{tabular}

\section{Relationship between Empirical Scaling Factor and In Vivo Clearance}

To further characterize the underprediction of in vivo $\mathrm{CL}_{\mathrm{int}, \mathrm{u}}$ in human and rat hepatocytes and liver microsomes, ESFs were calculated for individual compounds in each system and species and plotted as a function of observed $\mathrm{CL}_{\text {int,u }}$ (Fig. 3). A similar trend of increasing ESF with increasing in vivo $\mathrm{CL}_{\text {int,u }}$ was observed for human and rat hepatocytes (Fig. 3, A and C), indicating clearance-dependent underprediction. For microsomes, clearance dependency was also apparent, with comparable magnitude between the two in vitro systems (Fig. 3, B and D).

Comparison of the average ESF between segregated levels of in vivo $\mathrm{CL}_{\mathrm{int}, \mathrm{u}}$ showed an exponential progression of underprediction of a similar magnitude between human hepatocytes and microsomes up to $10,000 \mathrm{ml} / \mathrm{min} / \mathrm{kg}$, despite the high variance (Table 3). Above $10,000 \mathrm{ml} / \mathrm{min} / \mathrm{kg}$, detailed comparison was not justified due to few individual data, although a lower limitation in prediction by hepatocytes compared with microsomes was apparent. For rat, there was also quantitative agreement between the systems - although, as for human, there was marginally less underprediction evident for microsomes compared with hepatocytes. In addition, there was a progression of underprediction similar to human, albeit out of step by an order of magnitude (in terms of observed $\mathrm{CL}_{\mathrm{int}, \mathrm{u}}$ subgroup), possibly reflecting species differences in metabolic rates (as noted previously). Together, these quantitative prediction profiles provide clear evidence of strong species-independent trends and, to a large extent, system-independent trends in clearance-dependent underprediction.

Examining the ESF trends in terms of drug type, as denoted by the BDDCS, there was extensive overlap between permeable drugs cleared by metabolism (class 1) and similar drugs that were potential hepatic uptake substrates and that might have their clearance limited by this (class 2) (Fig. 3). For human, the trend of increasing ESF with in vivo $\mathrm{CL}_{\text {int }}$ appeared to be independent of drug type, although this was clearer for hepatocytes compared with microsomes; in the latter system, a number of class 2 or 3 drugs were predicted accurately or overpredicted, possibly reflecting enhanced access to metabolic enzymes in this system (Fig. 3, A and B). A similar lack of drug-type dependency was evident also for rat, although a number of the most highly cleared drugs were potentially dependent on transport for clearance (classes 2-4). The drug type assignment according to BDDCS is necessarily imprecise and some of the drugs designated as BDDCS class 2 could be otherwise designated as dependent on metabolism, rather than transport, according to the alternative extended clearance classification system (Varma et al., 2015) by virtue of being neutral or basic compounds; this would include a considerable number of the proprietary compounds in the rat data sets. As such, there would be a predominance of compounds dependent on metabolic clearance. Comparing human with rat, the trend in increasing ESF with in vivo $\mathrm{CL}_{\text {int }}$ was generally independent of species and to a large extent, in vitro system.

Use of ESFs between in vitro and in vivo from preclinical species has been suggested as a pragmatic refinement for methodology for human prediction (Naritomi et al., 2003). In the current analysis, similarity in ESF between rat and human might appear to support this, at least in general terms. However, for those drugs common to both rat and human data sets ( $n=24$ for hepatocytes; $n=17$ for microsomes) there was no apparent correlation between the species for either system (data not shown), indicating a lack of drug dependency in the scaling factor and hence persistence of considerable uncertainty on an individual drug basis.

\section{Prediction of In Vivo Clearance of UGT Substrates}

Within the human hepatocyte data set, drugs subject to glucuronidation were categorized as high $(\geq 0.75)$, medium-high $(0.50-0.75)$, mediumlow $(0.25-0.50)$, or low $(<0.25)$ fraction metabolized by UGT. As a single group, glucuronidated drugs span a large range of in vivo $\mathrm{CL}_{\mathrm{int}, \mathrm{u}}$

TABLE 2

Accuracy and precision of in vivo $\mathrm{CL}_{\mathrm{int}, \mathrm{u}}$ predictions in fresh and cryopreserved human hepatocytes

Data represented by AFE, average fold underprediction, RMSE, and percentage of predictions that fall within, above, and below 2-fold of observed in vivo $C_{\text {Lint, }}$. Analyses of the complete data set and a reduced data set of common drugs only are presented; $n=$ number of compounds.

\begin{tabular}{lccccc}
\hline \multirow{2}{*}{ Parameter } & \multicolumn{2}{c}{ Complete Data Set } & & \multicolumn{2}{c}{ Common Drugs } \\
\cline { 2 - 3 } \cline { 5 - 6 } & Fresh & Cryopreserved & & Fresh & Cryopreserved \\
\hline$n$ & 52 & 93 & & 43 & 43 \\
AFE & 0.25 & 0.23 & & 0.25 & 0.22 \\
Average fold underprediction & 3.9 & 4.3 & & 4.0 & 4.5 \\
RMSE & 2891 & 3013 & & 390 & 395 \\
Predictions within 2-fold of observed (\%) & 29 & 22 & & 26 & 26 \\
Predictions more than 2-fold above observed (\%) & 8 & 6 & & 7 & 2 \\
Predictions more than 2-fold below observed (\%) & 63 & 72 & & 67 & 72 \\
\hline
\end{tabular}




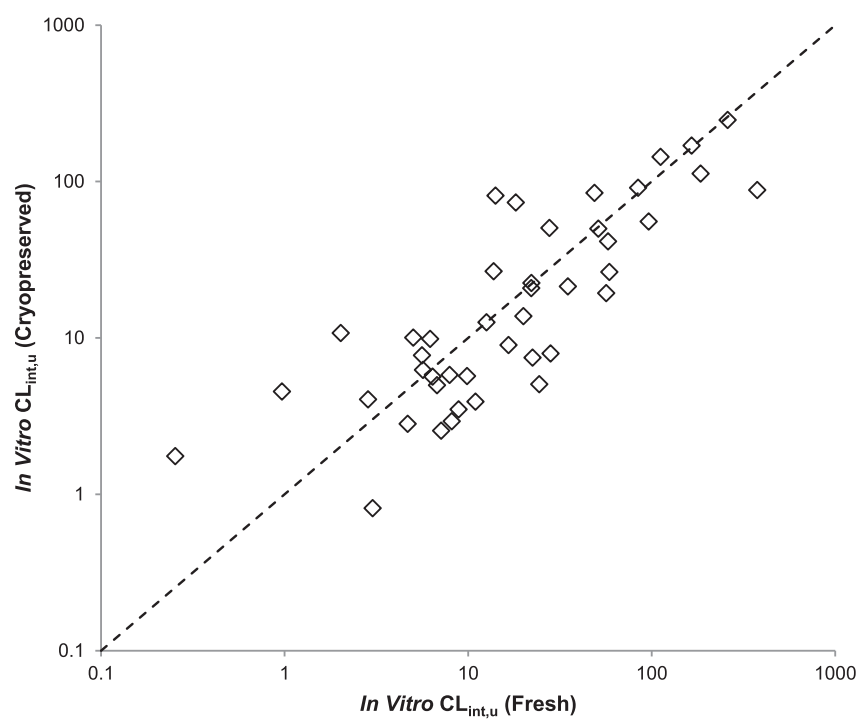

Fig. 2. Comparison of predicted in vivo $\mathrm{CL}_{\mathrm{int}, \mathrm{u}}$ in fresh and cryopreserved human hepatocytes (drugs common to both preparations only). Dashed line represents unity.

$(<10$ to $>1000 \mathrm{ml} / \mathrm{min} / \mathrm{kg}$ ) and show comparable underprediction to drugs metabolized predominantly by other enzymes (P450). There appears to be no relationship between the fraction metabolized by UGT and underprediction of in vivo $\mathrm{CL}_{\text {int,u }}$ (Fig. 4).

\section{Prediction of In Vivo Clearance of Acidic, Basic, and Neutral Drugs}

There was extensive overlap of prediction accuracy for drugs identified in groups according to ionic character for prediction made for both human and rat hepatocytes (Fig. 5) and hence this criterion was considered too simplistic for distinguishing prediction trends.

\section{Assessment of Extent of Underprediction in Relation to fu $u_{p}$ and BDDCS}

To assess the extent of any relationship between binding in blood (assumed to be mostly due to binding to plasma protein), ESF was plotted against $\mathrm{u}_{\mathrm{p}}$ for both human hepatocyte and microsome data sets (Fig. 6, A and B). Visually, there was no trend in prediction accuracy with $\mathrm{fu}_{\mathrm{p}}$ for either data set, although there was a clear tendency for BDDCS class 2 drugs to be more highly bound than those of class 1, as highlighted by Bowman and Benet (2016). The equivalent relationship for total blood binding $\left(\mathrm{fu}_{\mathrm{b}}\right)$ was also examined; however, since there were only very minor differences (data not shown) this was not analyzed further.

Segregating the drugs by level of $\mathrm{fu}_{\mathrm{p}}$ showed that the majority of drugs $(90 \%)$ had fu $\mathrm{p}_{\mathrm{p}}$ values either within the range of $0.01-0.1(40 \%)$ or $0.1-1(50 \%)$; the average ESF was between 3 and 4 for hepatocytes and between 2 and 3 for microsomes across these two levels of fu $\mathrm{p}_{\mathrm{p}}$ (Table 4). There were relatively few drugs with $\mathrm{fu}_{\mathrm{p}}$ values in the lower range of 0.001-0.01, and when excluding one/two highly aberrant values for hepatocytes and microsomes the ESF was about 6 (Table 4). For the drugs of BDDCS class 1 specifically, the ESF was between 3 and 6 for $\mathrm{fu}_{\mathrm{p}}$ across the ranges of $0.01-0.1$ and $0.1-1$ for hepatocytes, and between 2 and 4 across the same ranges for microsomes (Table 4). For the same $\mathrm{fu}_{\mathrm{p}}$ ranges, the ESF for class 2 drugs was between 4 and 13 for hepatocytes and between 1 and 3 for microsomes (Table 4$)$. The greater ESF values obtained for the lowest fu $u_{p}$ level for all drugs $(6.0$ and 5.6 for hepatocytes and microsomes, respectively) were largely reflected in class 2 drugs (8.2 and 6.3). A single compound in the microsome data set was identified as BDDCS class 4 but was excluded from the tabular analysis. Taken together, these observations indicate a lack of relationship between $\mathrm{fu}_{\mathrm{p}}$ and prediction accuracy and have no bearing on the trend of clearance-dependent prediction described previously.

It is recognized that some historical fu $\mathrm{p}$ values may be biased by lack of assay pH control (Kochansky et al., 2008). An adjustment of fu values for bases (average 2.6-fold increase, Kochansky et al., 2008) in this data set would cause some skew toward overall greater $\mathrm{fu}_{\mathrm{p}}$ for the values in the range of 0.1-1 due to the relatively weak binding of these compounds; however, exclusion of bases would not impact the lack of relationship between $\mathrm{fu}_{\mathrm{p}}$ and ESF for acidic and neutral drugs. Because the data cannot be retrospectively corrected with respect to exact assay conditions, such an adjustment was not made.

\section{Current Status of In Vitro Prediction of Clearance}

Prediction of clearance is a key component of drug discovery. However, as this report and many others verify, in vitro measured $\mathrm{CL}_{\text {int }}$ (using human/rat hepatocytes/hepatic microsomes) extrapolated to in vivo is not reliably quantitative on a physiologically mechanistic basis; a general problem of some 2- to 5-fold underprediction has been increasingly recognized for more than a decade (Shibata et al., 2002; Hallifax et al., 2005; Ito and Houston, 2005; Riley et al., 2005; Brown et al., 2007; Stringer et al., 2008). Although this problem can be circumvented by empirical corrections based on available data sets across a range of drugs, as has been reported (Sohlenius-Sternbeck et al., 2012; Yamagata et al., 2017), a high level of uncertainty remains for any individual case. This situation cannot be resolved without thorough understanding of the underlying causes of bias and imprecision that characterize prediction of clearance, as highlighted in a report by Bowman and Benet (2016).

The use of in vitro systems has progressed from microsomes to hepatocytes and from the rat preclinical species to human over several decades; however, recognition of the performance and limitations of component processes, let alone the causes of underprediction, has been slow. Hallifax et al. (2010) showed that predictions from human hepatocytes tended to be increasingly poorer with increasing in vivo $\mathrm{CL}_{\text {int }}$; a less distinct trend was observed for HLM, indicating that the intact cell system was inherently unsuitable for handling highly cleared drugs. Whether this implication was specific to human hepatocytes was not resolvable due to the paucity of reported data for rat at that time. However, the position has changed regarding studies in rat, and a viable comparison with human has become realistic and hence a key component of this commentary.

This current assessment shows an average underprediction of in vivo $\mathrm{CL}_{\text {int }}$ by human hepatocytes $(n=101)$ of 4.2 -fold, which confirms the bias reported by Hallifax et al. (2010) $(n=89)$. However, in addition, this study shows a similar (4.7-fold) underprediction from rat hepatocytes $(n=128)$, and in both cases despite the anticipated imprecision there is a strong trend of clearance dependency in prediction accuracy. Quantitatively, prediction accuracy ranges from unbiased (approximately 1-fold) for drug clearance below $10 \mathrm{ml} / \mathrm{min} / \mathrm{kg}$ in vivo $\mathrm{CL}_{\text {int }}$ to about either 20 -fold (rat) or 30-fold (human) underprediction for drug clearance between 1000 and $10,000 \mathrm{ml} / \mathrm{min} / \mathrm{kg}$. Since their initial use in clearance experiments, it has been accepted that human hepatocytes have been inherently more variable and subjected to more preparative and experimental variation than their rat counterparts; an additional caveat has been the possible mismatch between enzyme activity in donors and healthy volunteers and clinical patients, with consequential uncertainties (Iwatsubo et al., 1996, 1997; Wilson et al., 2003; Ito and Houston, 2004, 2005; Riley et al., 2005; Hallifax and Houston, 2009). By contrast, early 

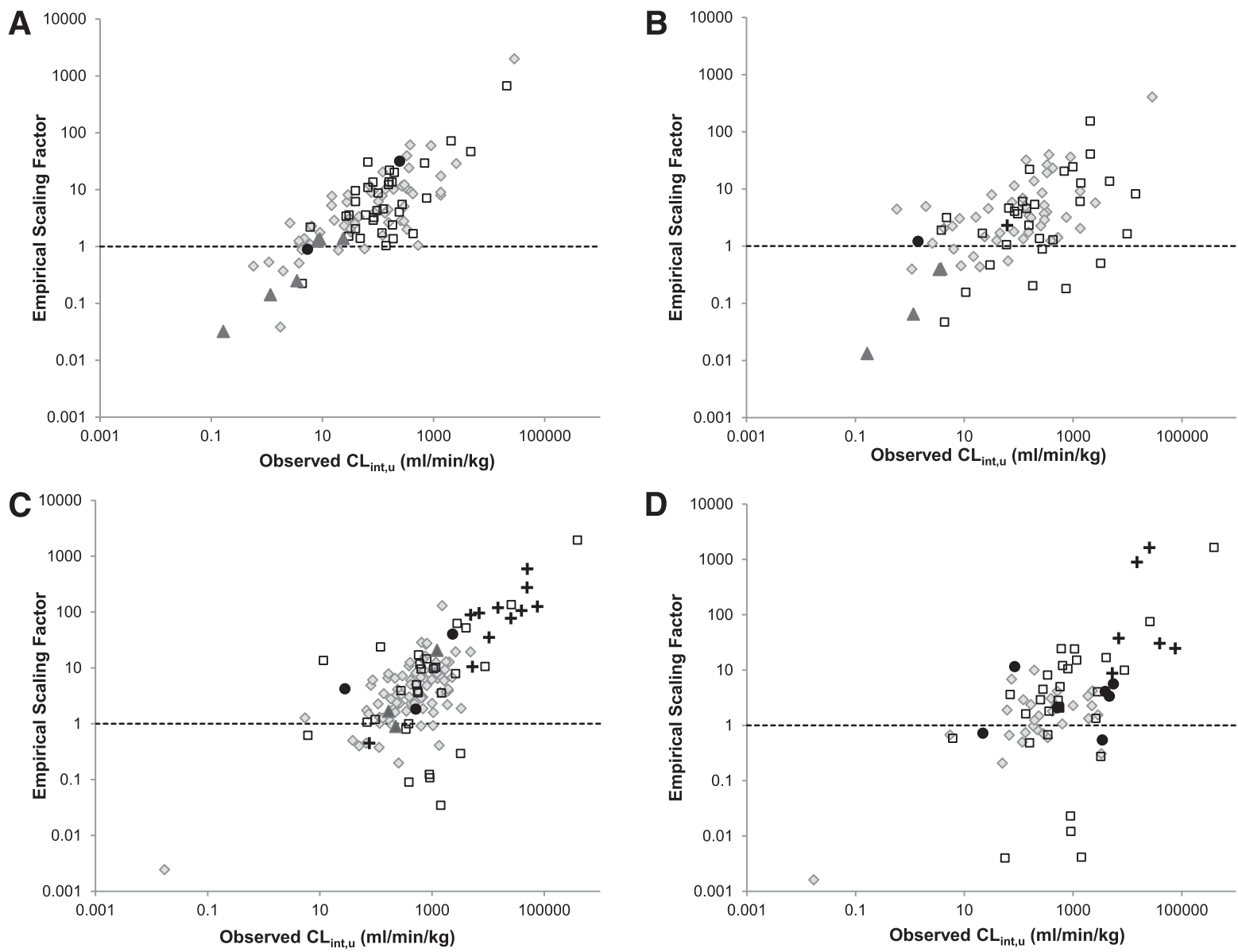

Fig. 3. Relationship between the ESF and observed $\mathrm{CL}_{\text {int,u }}$ according to BDDCS classification for hepatocytes $(\mathrm{A}$ and $\mathrm{C})$ and microsomes $(\mathrm{B}$ and $\mathrm{D})$ in human $(\mathrm{A}$ and $\mathrm{B})$ and rat $(\mathrm{C}$ and D). Compounds are represented as BDDCS class $1(\vartheta)$, class $2(\square)$, class $3(\Delta)$, class $4(+)$, or unclassified $(\bullet)$. BDDCS assignments for propriety compounds $(n=$ 88, rat hepatocytes; $n=37$ rat microsomes) were judged on available physicochemical information provided with source publications.

rat hepatocyte studies indicated a lack of bias (Houston and Carlile, 1997), and consequently underprediction of clearance has been considered specific to the human situation. Now, using accumulated data from over a decade, we have shown that this is not the case and that clearance-dependent prediction bias is effectively species independent, implying that the methodology for determining $\mathrm{CL}_{\text {int }}$ in hepatocytes is inadequate specifically for moderate to highly cleared drugs.

In microsomes, the average underprediction of in vivo $\mathrm{CL}_{\text {int }}$ was less than that in hepatocytes for both species, at 2.3-fold for rat $(n=71)$ and 2.8 -fold for human $(n=83)$, although the proportion of predictions within 2-fold was similar to hepatocytes. However, as for hepatocytes, there was a clear trend of clearance dependency in prediction accuracy with a similar exponential slope of increasing underprediction with in vivo $\mathrm{CL}_{\text {int }}$. Both of these findings - extent of average underprediction and clearance dependency in microsomes-were unexpected. Previously, underprediction from microsomes has been reported as greater than from hepatocytes and seemingly explained by absence of additional potentially critical clearance pathways (Engtrakul et al., 2005; Riley et al., 2005; Hallifax et al., 2010). Based on the present, most extensive study to date, it must be considered that the negative consequences of selectivity of enzyme pathways in microsomes is offset by other factors such as removal of ratelimiting access of drug to metabolic enzymes. Furthermore, the BDDCS

TABLE 3

Average individual ESF for predicted $\mathrm{CL}_{\mathrm{int}, \mathrm{u}}$ according to level of observed $\mathrm{CL}_{\mathrm{int}, \mathrm{u}}$ for human and rat hepatocytes and liver microsomes

\begin{tabular}{|c|c|c|c|c|c|c|c|c|}
\hline \multirow{4}{*}{ Observed $\mathrm{CL}_{\mathrm{int}, \mathrm{u}}$} & \multicolumn{8}{|c|}{ ESF } \\
\hline & \multicolumn{4}{|c|}{ Human } & \multicolumn{4}{|c|}{ Rat } \\
\hline & \multicolumn{2}{|c|}{ Hepatocytes } & \multicolumn{2}{|c|}{ Microsomes } & \multicolumn{2}{|c|}{ Hepatocytes } & \multicolumn{2}{|c|}{ Microsomes } \\
\hline & Log Average & $n$ & Log Average & $n$ & Log Average & $n$ & Log Average & $n$ \\
\hline \multicolumn{9}{|l|}{$\mathrm{ml} / \mathrm{min} / \mathrm{kg}$} \\
\hline$<10$ & 0.61 & 21 & 0.70 & 17 & 0.13 & 3 & 0.086 & 3 \\
\hline $10-100$ & 3.9 & 32 & 1.8 & 20 & 1.6 & 12 & 0.83 & 8 \\
\hline $100-1000$ & 7.1 & 40 & 4.6 & 34 & 3.2 & 67 & 1.7 & 34 \\
\hline $1000-10,000$ & 22 & 6 & 7.5 & 10 & 7.2 & 37 & 2.5 & 20 \\
\hline$>10,000$ & 1200 & 2 & 58 & 2 & 180 & 9 & 230 & 6 \\
\hline
\end{tabular}




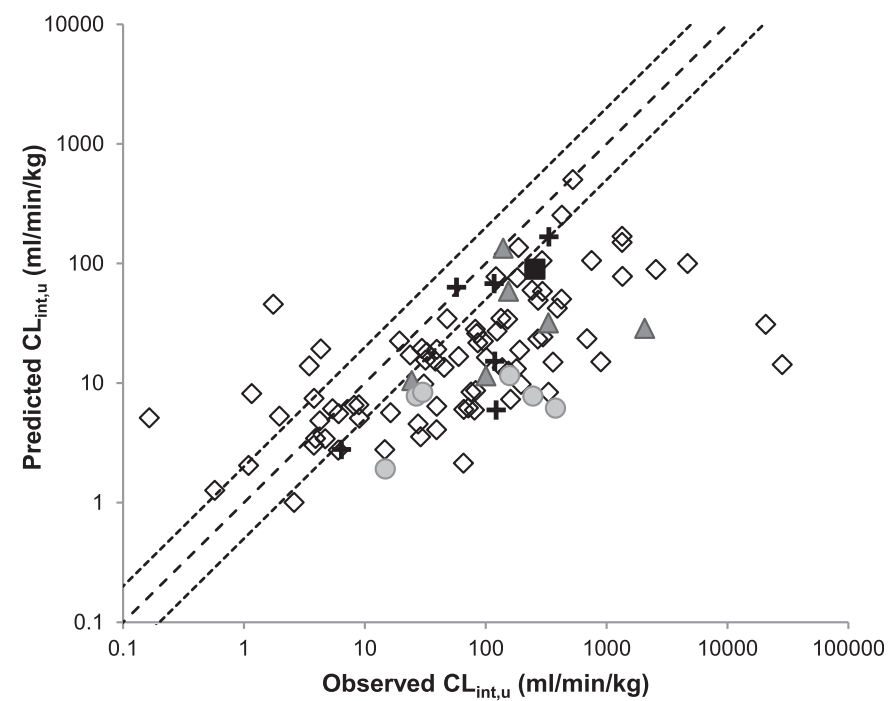

Fig. 4. Comparison of predicted $\mathrm{CL}_{\text {int,u }}$ in human hepatocytes with observed $C \mathrm{~L}_{\text {int,u }}$. Drugs subject to glucuronidation are identified as high $(\geq 0.75)(\odot)$, medium high

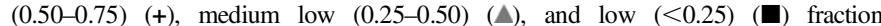
metabolized by UGT; all other compounds are represented as $(\diamond)$. Dashed line represents unity and dotted lines represent a 2 -fold margin of error.

analysis indicates that the trends in bias were apparently independent of the importance of clearance mechanism. Clearance-dependent prediction bias of a similar magnitude by both cellular and subcellular systems suggests influential factors beyond those previously mentioned.

Aside from experimental limitations, potential problems within the physiologic scaling methodology remain pertinent to this analysis. Here, as in most studies, the predictions used the well-stirred liver model because the parallel tube liver model (at the other extreme of drug hepatic dispersion) offered only marginal reduction in bias and precision (data not shown), consolidating previous conclusions (Hallifax et al., 2010). The discussion is necessarily limited to conventional non-permeabilitylimited models of hepatic clearance for two main reasons: the historical predominance of drugs not limited by permeability in their hepatic uptake and the lack of distinction of nonmetabolic rate-limiting processes within the available in vitro data. Extended liver clearance models may be appropriate for an increasing number of drugs due to reliance on uptake transport, and indeed lack of in vitro distinction of such processes has been suggested as a reason for underprediction of clearance (Chiba et al.,

A

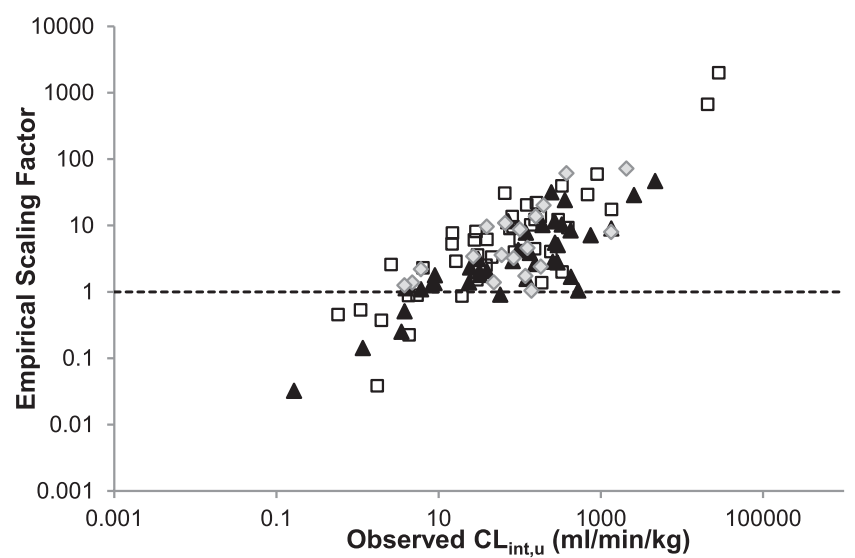

2009; Patilea-Vrana and Unadkat, 2016; Korzekwa and Nagar, 2017). However, this report attempts to focus on more general prediction trends. Beyond liver model selection, binding of drug to plasma protein is another important physiologic factor in prediction methodology that has been reappraised recently. The fu $u_{b}$ value is potentially highly influential in determining in vivo $\mathrm{CL}_{\text {int }}$ from clearance, especially where the $\mathrm{fu}_{\mathrm{b}}$ value is low $(<0.05)$; the extent of binding within blood, or at least to plasma proteins, has been reported to correlate with underprediction (Soars et al., 2007b; Ring et al., 2011). There has been a perception that the $\mathrm{fu}_{\mathrm{p}}$ value is difficult to measure at or below about 0.05 and this may be seen as a source of imprecision if not also bias. Although Riccardi et al. (2015) demonstrated that such low levels can be measured accurately with sufficiently controlled experiments the historical data might still be questioned. In addition, it has been shown that historical data might be erroneous where assay $\mathrm{pH}$ was not adequately controlled (Kochansky et al., 2008). This would appear to affect the $\mathrm{fu}_{\mathrm{p}}$ value of basic compounds more than others, although basic drugs show no bias in prediction relative to acids and neutrals. There is some lack of agreement in the literature on whether extensive binding is related to poor prediction of clearance; Hallifax and Houston (2012) demonstrated a lack of relation between binding and prediction based on analysis within the database reported in 2010, in contrast to earlier reports (Chiba et al., 2009). More recently, Bowman and Benet (2016) found that drugs categorized as highly bound tended to have greater prediction bias than lesser bound drugs, particularly for BDDCS class 2; however, their analysis used subgroups of relatively small numbers. The present study, examining trends among the entire data sets for these most recent databases (human hepatocytes and microsomes), appears not to support substantial involvement of the degree of binding to plasma proteins. Alternatives to fraction unbound, i.e., mechanistic involvement of bound drug in uptake (Poulin et al., 2012), have been discussed elsewhere (Hallifax and Houston, 2012) and are beyond the scope of this report.

Preparative experimental steps, such as cryopreservation of human hepatocytes, have been seen as potentially detrimental; however, within the human hepatocyte data set in this study predictions using cryopreserved hepatocytes were not biased compared with predictions from freshly isolated hepatocytes-focusing attention on downstream experimental procedures. This consolidates previous reports by Diener et al. (1995), Li et al. (1999), Hewitt et al. (2000), Lau et al. (2002), Naritomi et al. (2003), McGinnity et al. (2004), Blanchard et al. (2005), and Floby et al. (2009). For hepatocyte assays, false clearance prediction of drugs that rely on uptake transport might be expected either due to unrepresentative

\section{B}

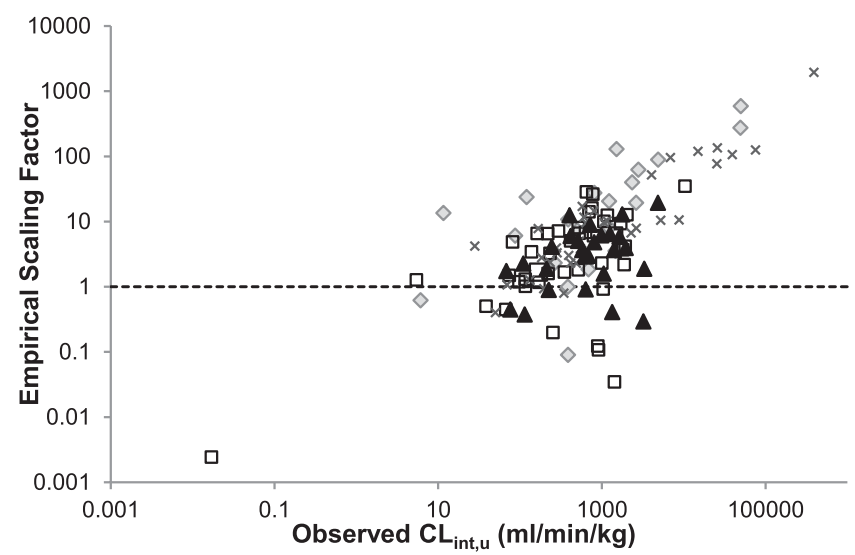

Fig. 5. Relationship between the ESF and observed $\mathrm{CL}_{\mathrm{int}, \mathrm{u}}$ according to the drug ionic character for human (A) and rat (B) hepatocytes. Compounds are represented as acidic ( ) , basic $(\boldsymbol{\Lambda})$, neutral $(\square)$, or unclassified (x). 
A

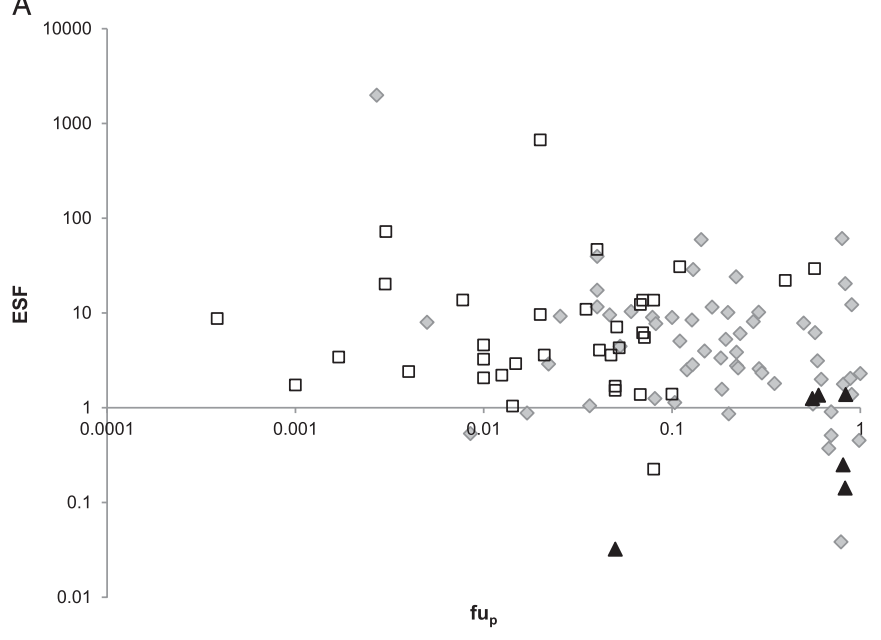

B

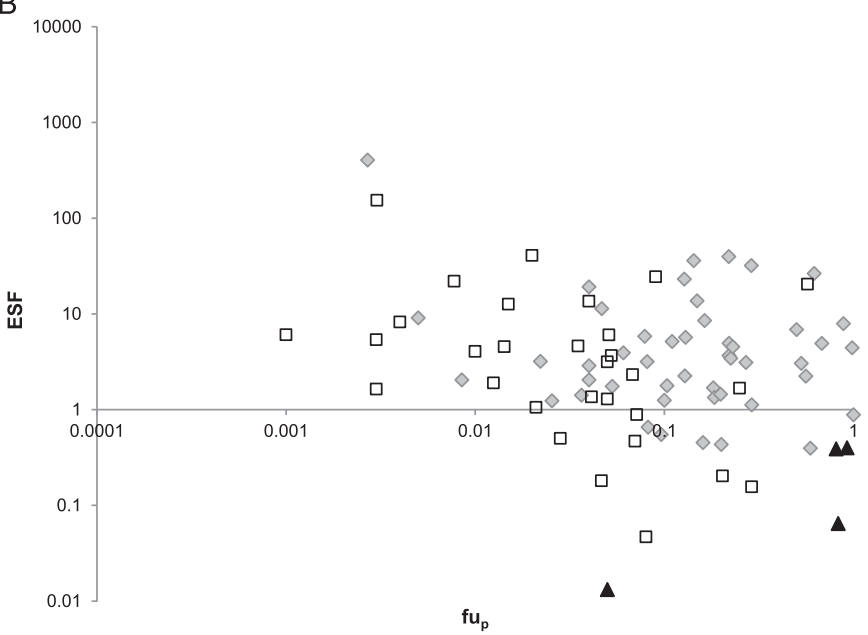

Fig. 6. Relationship between the $\mathrm{fu}_{\mathrm{p}}, \mathrm{ESF}$, and BDDCS class in (A) human hepatocytes and (B) HLM. Drugs are represented as BDDCS class 1() , class $2(\square)$, and class $3(\mathbf{\Delta})$.

transporter activity in vitro (Soars et al., 2007a) or absolute loss of activity due to instability in vitro. In the data sets presented here, the impact of such substrates on the trend in prediction bias appears to be limited; the majority of drugs included are considered to be cleared predominantly by metabolic (particularly $\mathrm{P} 450$ ) enzymes without rate limitation by uptake transport. Those compounds considered liable to transport dependence largely followed the same trend, which was similar in magnitude between hepatocytes and microsomes (for both human and rat). Predictions for drugs identified as UGT substrates within the hepatocyte data set were not clearly resolved from others, again indicating no particular hepatocyte bias over microsomes for these pathways.

\section{Future Prospects to Refine In Vitro Prediction of Clearance}

A number of factors can be speculated to cause the clearance-dependent underprediction now established in both human and rat hepatocytes and microsomes. The increase in prediction bias seen with increasing in vivo $\mathrm{CL}_{\text {int }}$ has been previously speculated as due to an increasing incidence of permeation rate limitation among high-turnover drugs (Hallifax et al., 2010). This would be supported by the similar trend in the present study, which involves many drugs not limited by active uptake. However, Hallifax et al. (2012) showed no discernible relationship between prediction accuracy and either of several independent measures of passive permeability for a wide range of drugs. Huang et al. (2010) showed that among a number of highly
TABLE 4

Average individual ESF for predicted $\mathrm{CL}_{\mathrm{int}, \mathrm{u}}$ according to $\mathrm{fu}_{\mathrm{p}}$ and BDDCS classification for human hepatocytes and liver microsomes

Numbers in italic indicate the exclusion of a single compound in this class; numbers in bold indicate exclusion of two compounds from this group.

\begin{tabular}{|c|c|c|c|c|c|c|}
\hline \multirow{3}{*}{ Category } & \multicolumn{6}{|c|}{ ESF } \\
\hline & \multicolumn{2}{|c|}{$0.001-0.01 \mathrm{fu}_{\mathrm{p}}$} & \multicolumn{2}{|c|}{$0.01-0.1 \mathrm{fu}_{\mathrm{p}}$} & \multicolumn{2}{|l|}{$0.1-1 \mathrm{fu}_{\mathrm{p}}$} \\
\hline & Log Average & $n$ & Log Average & $n$ & Log Average & $n$ \\
\hline \multicolumn{7}{|l|}{ Hepatocytes } \\
\hline Total & 6.0 & 9 & 4.0 & 37 & 3.3 & 53 \\
\hline \multicolumn{7}{|c|}{ Hepatocytes (BDDCS) } \\
\hline Class 1 & 2.1 & 2 & 5.8 & 13 & 3.5 & 42 \\
\hline Class 2 & 8.2 & 7 & 4.1 & 23 & 13 & 4 \\
\hline Class 3 & & 0 & 0.032 & 1 & 0.61 & 5 \\
\hline \multicolumn{7}{|l|}{ Microsomes } \\
\hline Total & 5.6 & 7 & 2.1 & 35 & 2.6 & 39 \\
\hline \multicolumn{7}{|c|}{ Microsomes (BDDCS) } \\
\hline Class 1 & 4.3 & 2 & 2.7 & 13 & 3.8 & 31 \\
\hline Class 2 & 6.3 & 5 & 2.3 & 20 & 1.0 & 4 \\
\hline Class 3 & & 0 & 0.013 & 1 & 0.22 & 3 \\
\hline
\end{tabular}

permeable proprietary compounds, those that were also efflux substrates gave greater underprediction. Umehara and Camenisch (2012) suggested using a combination of hepatocytes and microsome assays to distinguish where clearance was rate limited by permeation. While the phenomenon of permeation rate limitation to hepatic clearance in vitro may nevertheless occur, the present analysis provides no definite evidence of a permeation rate limitation, and indeed the similar trend in underprediction with increasing clearance between hepatocytes and microsomes suggests involvement of an alternative, common explanation.

Cofactor depletion has been suggested as a possible cause of clearance-dependent underprediction when using hepatocytes with high-turnover drugs (Swales et al., 1996; Swales and Utesch, 1998; Steinberg et al., 1999; Hengstler et al., 2000; Hewitt et al., 2000; Hewitt and Utesch, 2004; Hallifax et al., 2010; Foster et al., 2011). However, this would not explain the similar trends in underprediction between hepatocytes and microsomes without considerable coincidence, given the different media used (cell culture medium versus NADPH or regenerating system). Cofactor depletion has been a suggested consequence of cryopreservation, and human hepatocytes (often cryopreserved) tend to be used with lower viability (typically, not exceeding $80 \%-90 \%$ in the studies included here, where reported) than rat hepatocytes, implying greater potential loss of cofactors in these preparations (potentially reflected in cells deemed viable or not). However, overall, the lack of any prediction bias among numerous studies for cells prepared this way does not support a significant role for cofactor loss associated with cryopreservation.

For typical drug depletion assays, suboptimal substrate concentration would be a source of error (imprecision) among predictions, and if there was a tendency to use concentrations approaching or greater than the $K_{\mathrm{M}}$ values for key pathways this might manifest as a source of bias. In a review by Klopf and Worboys (2010), the widely practiced experimental expediency of a fixed substrate concentration of the order of $1 \mu \mathrm{M}$ for all drugs screened for metabolic stability was highlighted. For some drugs, this practice might exclude otherwise influential high-affinity, lowcapacity enzyme sites with resultant poor predictions. Highly metabolically cleared drugs are often substrates of the CYP2D and CYP3A families, in which $K_{\mathrm{M}}$ values at around or below $1 \mu \mathrm{M}$ are not uncommon (Lewis and Ito, 2010); such cases, if ignored, could lead to clearance-dependent prediction.

The phenomenon of the unstirred water layer has been extensively discussed with respect to intestinal permeability in vitro systems (Karlsson and Artursson, 1991; Naruhashi et al., 2003; Avdeef et al., 
2004; Korjamo et al., 2009) but has been little discussed with regard to metabolic clearance methodology, possibly due to segregation of drug discovery functions within companies. However, the unstirred water layer should at least be considered for hepatocyte assays, considering the present problem with high turnover drugs. Diffusion through the unstirred water layer might be rate limiting for such compounds, although, again, a degree of coincidence between the external environment of the whole cell and that of its microsomal fraction would be implied.

Ultimately, the in vitro causes of underprediction are likely to be multifactorial. Further investigation into possible experimental causes of underprediction of clearance-and its clearance dependence-is highly desirable in the light of the findings of this report. As the trend toward larger molecules with multiple charges and increasing involvement of dispositional transporters continues, more variable factors are added to the situation. While a broader view of the role of transporters and their interrelationship with enzymes will be important, detailed and systematic investigations need to focus on certain other fundamental factors such as the passive permeation mechanism, integrity of membrane and unstirred water barriers, and their relative impact on rates. Other basic assay parameters such as substrate concentration dependence in $\mathrm{CL}_{\text {int }}$ also need to be considered. Some of these potentially instrumental phenomena have recently been investigated in our laboratory and the findings will be reported in due course.

\section{Acknowledgments}

We thank James Harrison for useful contributions to the database compilation.

\section{Authorship Contributions}

Participated in research design: Wood, Hallifax, Houston.

Performed data analysis: Wood, Hallifax.

Wrote or contributed to the writing of the manuscript: Wood, Hallifax, Houston.

\section{References}

Akabane T, Gerst N, Masters JN, and Tamura K (2012a) A quantitative approach to hepatic clearance prediction of metabolism by aldehyde oxidase using custom pooled hepatocytes. Xenobiotica 42:863-871.

Akabane T, Gerst N, Naritomi Y, Masters JN, and Tamura K (2012b) A practical and direct comparison of intrinsic metabolic clearance of several non-CYP enzyme substrates in freshly isolated and cryopreserved hepatocytes. Drug Metab Pharmacokinet 27:181-191.

Avdeef A, Nielsen PE, and Tsinman O (2004) PAMPA—a drug absorption in vitro model 11 Matching the in vivo unstirred water layer thickness by individual-well stirring in microtitre plates. Eur J Pharm Sci 22:365-374.

Bayliss MK, Bell JA, Jenner WN, Park GR, and Wilson K (1999) Utility of hepatocytes to model species differences in the metabolism of loxtidine and to predict pharmacokinetic parameters in rat, dog and man. Xenobiotica 29:253-268.

Benet LZ, Broccatelli F, and Oprea TI (2011) BDDCS applied to over 900 drugs. AAPS J 13: 519-547.

Blanchard N, Alexandre E, Abadie C, Lavé T, Heyd B, Mantion G, Jaeck D, Richert L, and Coassolo $\mathrm{P}$ (2005) Comparison of clearance predictions using primary cultures and suspensions of human hepatocytes. Xenobiotica 35:1-15.

Bowman CM and Benet LZ (2016) Hepatic clearance predictions from in vitro-in vivo extrapolation and the biopharmaceutics drug disposition classification system. Drug Metab Dispos 44: 1731-1735.

Brown HS, Griffin M, and Houston JB (2007) Evaluation of cryopreserved human hepatocytes as an alternative in vitro system to microsomes for the prediction of metabolic clearance. Drug Metab Dispos 35:293-301

Chan TS, Yu H, Moore A, Khetani SR, and Tweedie D (2013) Meeting the challenge of predicting hepatic clearance of compounds slowly metabolized by cytochrome P450 using a novel hepatocyte model, HepatoPac. Drug Metab Dispos 41:2024-2032.

Chiba M, Ishii Y, and Sugiyama Y (2009) Prediction of hepatic clearance in human from in vitro data for successful drug development. AAPS J 11:262-276.

Cubitt HE, Houston JB, and Galetin A (2009) Relative importance of intestinal and hepatic glucuronidation-impact on the prediction of drug clearance. Pharm Res 26:1073-1083.

Davies B and Morris T (1993) Physiological parameters in laboratory animals and humans. Pharm Res 10:1093-1095.

De Buck SS, Sinha VK, Fenu LA, Nijsen MJ, Mackie CE, and Gilissen RAHJ (2007) Prediction of human pharmacokinetics using physiologically based modeling: a retrospective analysis of 26 clinically tested drugs. Drug Metab Dispos 35:1766-1780.

Diener B, Abdel-Latif H, Arand M, and Oesch F (1995) Xenobiotic metabolizing enzyme activities and viability are well preserved in EDTA-isolated rat liver parenchymal cells after cryopreservation. Toxicol Appl Pharmacol 130:149-153.

Engtrakul JJ, Foti RS, Strelevitz TJ, and Fisher MB (2005) Altered AZT ( $3^{\prime}$-azido- $3^{\prime}$-deoxythymidine) glucuronidation kinetics in liver microsomes as an explanation for underprediction of in vivo clearance: comparison to hepatocytes and effect of incubation environment. Drug Metab Dispos 33:1621-1627.

Floby E, Johansson J, Hoogstraate J, Hewitt NJ, Hill J, and Sohlenius-Sternbeck AK (2009) Comparison of intrinsic metabolic clearance in fresh and cryopreserved human hepatocytes. Xenobiotica 39:656-662.

Foster JA, Houston JB, and Hallifax D (2011) Comparison of intrinsic clearances in human liver microsomes and suspended hepatocytes from the same donor livers: clearance-dependent relationship and implications for prediction of in vivo clearance. Xenobiotica 41:124-136.

Gertz M, Harrison A, Houston JB, and Galetin A (2010) Prediction of human intestinal first-pass metabolism of 25 CYP3A substrates from in vitro clearance and permeability data. Drug Metab Dispos 38:1147-1158.

Hakooz N, Ito K, Rawden H, Gill H, Lemmers L, Boobis AR, Edwards RJ, Carlile DJ, Lake BG, and Houston JB (2006) Determination of a human hepatic microsomal scaling factor for predicting in vivo drug clearance. Pharm Res 23:533-539.

Hallifax D, Foster JA, and Houston JB (2010) Prediction of human metabolic clearance from in vitro systems: retrospective analysis and prospective view. Pharm Res 27:2150-2161.

Hallifax D, Galetin A, and Houston JB (2008) Prediction of metabolic clearance using fresh human hepatocytes: comparison with cryopreserved hepatocytes and hepatic microsomes for five benzodiazepines. Xenobiotica 38:353-367.

Hallifax D and Houston JB (2006) Binding of drugs to hepatic microsomes: comment and assessment of current prediction methodology with recommendation for improvement. Drug Metab Dispos 34:724-726 (author reply p 727).

Hallifax D and Houston JB (2009) Methodological uncertainty in quantitative prediction of human hepatic clearance from in vitro experimental systems. Curr Drug Metab 10:307-321.

Hallifax D and Houston JB (2012) Evaluation of hepatic clearance prediction using in vitro data: emphasis on fraction unbound in plasma and drug ionisation using a database of 107 drugs. J Pharm Sci 101:2645-2652.

Hallifax D, Rawden HC, Hakooz N, and Houston JB (2005) Prediction of metabolic clearance using cryopreserved human hepatocytes: kinetic characteristics for five benzodiazepines. Drug Metab Dispos 33:1852-1858.

Hallifax D, Turlizzi E, Zanelli U, and Houston JB (2012) Clearance-dependent underprediction of in vivo intrinsic clearance from human hepatocytes: comparison with permeabilities from artificia membrane (PAMPA) assay, in silico and caco-2 assay, for 65 drugs. Eur J Pharm Sci 45:570-574.

Hengstler JG, Utesch D, Steinberg P, Platt KL, Diener B, Ringel M, Swales N, Fischer T, Biefang K, Gerl $\mathrm{M}$, et al. (2000) Cryopreserved primary hepatocytes as a constantly available in vitro model for the evaluation of human and animal drug metabolism and enzyme induction. Drug Metab Rev 32:81-118.

Hewitt NJ, Fischer T, Zuehlke U, Oesch F, and Utesch D (2000) Metabolic activity of fresh and cryopreserved cynomolgus monkey (Macaca fascicularis) hepatocytes. Xenobiotica 30:665-681.

Hewitt NJ and Utesch D (2004) Cryopreserved rat, dog and monkey hepatocytes: measurement of drug metabolizing enzymes in suspensions and cultures. Hum Exp Toxicol 23:307-316.

Hosey CM, Chan R, and Benet LZ (2016) BDDCS predictions, self-correcting aspects of BDDCS assignments, BDDCS assignment corrections, and classification for more than 175 additional drugs. AAPS J 18:251-260.

Houston JB (1994) Utility of in vitro drug metabolism data in predicting in vivo metabolic clearance. Biochem Pharmacol 47:1469-1479.

Houston JB and Carlile DJ (1997) Prediction of hepatic clearance from microsomes, hepatocytes, and liver slices. Drug Metab Rev 29:891-922.

Huang L, Berry L, Ganga S, Janosky B, Chen A, Roberts J, Colletti AE, and Lin MHJ (2010) Relationship between passive permeability, efflux, and predictability of clearance from in vitro metabolic intrinsic clearance. Drug Metab Dispos 38:223-231.

Ito $\mathrm{K}$ and Houston JB (2004) Comparison of the use of liver models for predicting drug clearance using in vitro kinetic data from hepatic microsomes and isolated hepatocytes. Pharm Res 21:785-792.

Ito K and Houston JB (2005) Prediction of human drug clearance from in vitro and preclinical data using physiologically based and empirical approaches. Pharm Res 22:103-112.

Iwatsubo T, Hirota N, Ooie T, Suzuki H, Shimada N, Chiba K, Ishizaki T, Green CE, Tyson CA, and Sugiyama Y (1997) Prediction of in vivo drug metabolism in the human liver from in vitro metabolism data. Pharmacol Ther 73:147-171.

Iwatsubo T, Hirota N, Ooie T, Suzuki H, and Sugiyama Y (1996) Prediction of in vivo drug disposition from in vitro data based on physiological pharmacokinetics. Biopharm Drug Dispos 17:273-310.

Jones HM, Chen Y, Gibson C, Heimbach T, Parrott N, Peters SA, Snoeys J, Upreti VV, Zheng M, and Hall SD (2015) Physiologically based pharmacokinetic modeling in drug discovery and development: a pharmaceutical industry perspective. Clin Pharmacol Ther 97:247-262.

Jones HM and Houston JB (2004) Substrate depletion approach for determining in vitro metabolic clearance: time dependencies in hepatocyte and microsomal incubations. Drug Metab Dispos 32:973-982.

Kaiser G, Ackermann R, Dieterle W, Reimann IW, and Bieck PR (1992) Pharmacokinetics of the antidepressant levoprotiline after intravenous and peroral administration in healthy volunteers. Biopharm Drug Dispos 13:83-93.

Karlsson J and Artursson P (1991) A method for the determination of cellular permeability coefficients and aqueous boundary-layer thickness in monolayers of intestinal epithelial (Caco-2) cells grown in permeable filter chambers. Int J Pharm 71:55-64.

Kilford PJ, Gertz M, Houston JB, and Galetin A (2008) Hepatocellular binding of drugs: correction for unbound fraction in hepatocyte incubations using microsomal binding or drug lipophilicity data. Drug Metab Dispos 36:1194-1197.

Klopf W and Worboys P (2010) Scaling in vivo pharmacokinetics from in vitro metabolic stability data in drug discovery. Comb Chem High Throughput Screen 13:159-169.

Kochansky CJ, McMasters DR, Lu P, Koeplinger KA, Kerr HH, Shou M, and Korzekwa KR (2008) Impact of $\mathrm{pH}$ on plasma protein binding in equilibrium dialysis. Mol Pharm 5:438-448.

Korjamo T, Heikkinen AT, and Mönkkönen J (2009) Analysis of unstirred water layer in in vitro permeability experiments. J Pharm Sci 98:4469-4479.

Korzekwa K and Nagar S (2017) On the nature of physiologically-based pharmacokinetic model - a priori or a posteriori? mechanistic or empirical? Pharm Res 34:529-534.

Laethem ME, Lefebvre RA, Belpaire FM, Vanhoe HL, and Bogaert MG (1995) Stereoselective pharmacokinetics of oxprenolol and its glucuronides in humans. Clin Pharmacol Ther 57: 419-424.

Lau YY, Sapidou E, Cui X, White RE, and Cheng KC (2002) Development of a novel in vitro model to predict hepatic clearance using fresh, cryopreserved, and sandwich-cultured hepatocytes. Drug Metab Dispos 30:1446-1454.

Lewis DFV and Ito Y (2010) Human CYPs involved in drug metabolism: structures, substrates and binding affinities. Expert Opin Drug Metab Toxicol 6:661-674. 
Li AP, Lu C, Brent JA, Pham C, Fackett A, Ruegg CE, and Silber PM (1999) Cryopreserved human hepatocytes: characterization of drug-metabolizing enzyme activities and applications in higher throughput screening assays for hepatotoxicity, metabolic stability, and drug-drug interaction potential. Chem Biol Interact 121:17-35.

McGinnity DF, Soars MG, Urbanowicz RA, and Riley RJ (2004) Evaluation of fresh and cryopreserved hepatocytes as in vitro drug metabolism tools for the prediction of metabolic clearance. Drug Metab Dispos 32:1247-1253.

Miners JO and Mackenzie PI (1991) Drug glucuronidation in humans. Pharmacol Ther 51: 347-369.

Naritomi Y, Terashita S, Kagayama A, and Sugiyama Y (2003) Utility of hepatocytes in predicting drug metabolism: comparison of hepatic intrinsic clearance in rats and humans in vivo and in vitro. Drug Metab Dispos 31:580-588.

Naritomi Y, Terashita S, Kimura S, Suzuki A, Kagayama A, and Sugiyama Y (2001) Prediction of human hepatic clearance from in vivo animal experiments and in vitro metabolic studies with liver microsomes from animals and humans. Drug Metab Dispos 29:1316-1324.

Naruhashi K, Tamai I, Li Q, Sai Y, and Tsuji A (2003) Experimental demonstration of the unstirred water layer effect on drug transport in Caco-2 cells. J Pharm Sci 92:1502-1508.

Obach RS (1999) Prediction of human clearance of twenty-nine drugs from hepatic microsomal intrinsic clearance data: an examination of in vitro half-life approach and nonspecific binding to microsomes. Drug Metab Dispos 27:1350-1359.

Paixão P, Gouveia LF, and Morais JAG (2010) Prediction of the in vitro intrinsic clearance determined in suspensions of human hepatocytes by using artificial neural networks. Eur $J$ Pharm Sci 39:310-321.

Patilea-Vrana G and Unadkat JD (2016) Transport vs. metabolism: what determines the pharmacokinetics and pharmacodynamics of drugs? Insights from the extended clearance model. Clin Pharmacol Ther 100:413-418.

Poulin P and Haddad S (2013) Toward a new paradigm for the efficient in vitro-in vivo extrapolation of metabolic clearance in humans from hepatocyte data. J Pharm Sci 102:3239-3251.

Poulin P, Kenny JR, Hop CECA, and Haddad S (2012) In vitro-in vivo extrapolation of clearance: modeling hepatic metabolic clearance of highly bound drugs and comparative assessment with existing calculation methods. J Pharm Sci 101:838-851.

Riccardi K, Cawley S, Yates PD, Chang C, Funk C, Niosi M, Lin J, and Di L (2015) Plasma protein binding of challenging compounds. J Pharm Sci 104:2627-2636.

Riley RJ, McGinnity DF, and Austin RP (2005) A unified model for predicting human hepatic, metabolic clearance from in vitro intrinsic clearance data in hepatocytes and microsomes. Drug Metab Dispos 33:1304-1311.

Ring BJ, Chien JY, Adkison KK, Jones HM, Rowland M, Jones RD, Yates JW, Ku MS, Gibson $\mathrm{CR}, \mathrm{He} \mathrm{H}$, et al. (2011) PhRMA CPCDC initiative on predictive models of human pharmacokinetics, part 3: comparative assessement of prediction methods of human clearance. J Pharm Sci 100:4090-4110.

Shibata Y, Takahashi H, Chiba M, and Ishii Y (2002) Prediction of hepatic clearance and availability by cryopreserved human hepatocytes: an application of serum incubation method. Drug Metab Dispos 30:892-896.
Soars MG, Burchell B, and Riley RJ (2002) In vitro analysis of human drug glucuronidation and prediction of in vivo metabolic clearance. J Pharmacol Exp Ther 301:382-390.

Soars MG, Grime K, Sproston JL, Webborn PJH, and Riley RJ (2007a) Use of hepatocytes to assess the contribution of hepatic uptake to clearance in vivo. Drug Metab Dispos 35:859-865. Soars MG, McGinnity DF, Grime K, and Riley RJ (2007b) The pivotal role of hepatocytes in drug discovery. Chem Biol Interact 168:2-15.

Sohlenius-Sternbeck AK, Afzelius L, Prusis P, Neelissen J, Hoogstraate J, Johansson J, Floby E, Bengtsson A, Gissberg O, Sternbeck J, et al. (2010) Evaluation of the human prediction of clearance from hepatocyte and microsome intrinsic clearance for 52 drug compounds. Xenobiotica 40:637-649.

Sohlenius-Sternbeck AK, Jones C, Ferguson D, Middleton BJ, Projean D, Floby E, Bylund J, and Afzelius L (2012) Practical use of the regression offset approach for the prediction of in vivo intrinsic clearance from hepatocytes. Xenobiotica 42:841-853.

Steinberg P, Fischer T, Kiulies S, Biefang K, Platt KL, Oesch F, Böttger T, Bulitta C, Kempf P, and Hengstler J (1999) Drug metabolizing capacity of cryopreserved human, rat, and mouse liver parenchymal cells in suspension. Drug Metab Dispos 27:1415-1422.

Stringer R (2006) Evaluation of higher-throughput in vitro methods for the reliable prediction of in vivo metabolic clearance in humans. PhD Thesis, School of Pharmacy and Pharmaceutical Science, University of Manchester, Manchester, United Kingdom.

Stringer R, Nicklin PL, and Houston JB (2008) Reliability of human cryopreserved hepatocytes and liver microsomes as in vitro systems to predict metabolic clearance. Xenobiotica 38:1313-1329.

Swales NJ, Johnson T, and Caldwell J (1996) Cryopreservation of rat and mouse hepatocytes. II. Assessment of metabolic capacity using testosterone metabolism. Drug Metab Dispos 24:1224-1230. Swales NJ and Utesch D (1998) Metabolic activity of fresh and cryopreserved dog hepatocyte suspensions. Xenobiotica 28:937-948.

Umehara K and Camenisch G (2012) Novel in vitro-in vivo extrapolation (IVIVE) method to predict hepatic organ clearance in rat. Pharm Res 29:603-617.

Varma MV, Steyn SJ, Allerton C, and El-Kattan AF (2015) Predicting clearance mechanism in drug discovery: extended clearance classification system (ECCS). Pharm Res 32:3785-3802.

Wilson ZE, Rostami-Hodjegan A, Burn JL, Tooley A, Boyle J, Ellis SW, and Tucker GT (2003) Inter-individual variability in levels of human microsomal protein and hepatocellularity per gram of liver. Br J Clin Pharmacol 56:433-440.

Yamagata T, Zanelli U, Gallemann D, Perrin D, Dolgos H, and Petersson C (2017) Comparison of methods for the prediction of human clearance from hepatocyte intrinsic clearance for a set of reference compounds and an external evaluation set. Xenobiotica 47:741-751.

Address correspondence to: D. Hallifax, Centre for Applied Pharmacokinetic Research, Division of Pharmacy and Optometry, School of Health Sciences, Faculty of Biology, Medicine and Health, University of Manchester, Manchester Academic Health Science Centre, Manchester M13 9PT, UK. E-mail: David. Hallifax@manchester.ac.uk 